\title{
High-power, short-duration ablation in the coronary sinus: clinical cases and preliminary observations on swine hearts
}

\author{
Chengming $\mathrm{Ma}^{1}$ (D) $\cdot$ Xiaomeng Yin ${ }^{1}$ (D) Y Yunlong Xia ${ }^{1} \cdot$ Jiao Sun $^{2} \cdot$ Shiyu Dai $^{1} \cdot$ Lianjun Gao $^{1} \cdot$ Xianjie Xiao $^{1}$. \\ Yuanjun Sun ${ }^{1} \cdot$ Rongfeng Zhang $^{1} \cdot$ Yingxue Dong $^{1} \cdot$ Zhongzhen Wang $^{1} \cdot$ Xiaohong Yu $^{1}$
}

Received: 17 November 2020 / Accepted: 7 April 2021 / Published online: 15 April 2021

(C) The Author(s) 2021

\begin{abstract}
Purpose Coronary sinus-related arrhythmias are common; however, it is difficult to perform radiofrequency (RF) ablation at these sites efficiently and safely. High-power, short-duration ablation (HPSD) is a proven alternative strategy for pulmonary vein isolation (PVI); whether it can be applied to ablation of the coronary sinus is unknown. The purpose of this preliminary study was to evaluate the feasibility and safety of HPSD ablation in the coronary sinus.

Methods Firstly, we demonstrated 4 clinical cases of 3 types of arrhythmias who had unsuccessful ablation with standard power initially, but received successful ablations with HPSD. Secondly, RF ablation was performed in the coronary sinus ostium (CSO) and middle cardiac vein (MCV) of 4 in vitro swine hearts. Two protocols were compared: HPSD ( $45 \mathrm{~W} / 5 \mathrm{~S} \times 5$ rounds) and a conventional strategy that used low-power, long-duration ablation (LPLD: $25 \mathrm{~W} / 10 \mathrm{~S} \times 5$ rounds). The total duration of HPSD protocol was $25 \mathrm{~s}$, and which of LPLD was $50 \mathrm{~s}$.

Results A total of 28 lesions were created. HPSD can produce longer, wider, deeper, and larger lesions than LPLD. This difference was more pronounced when the ablation was in the MCV. One instance of steam pop occurred during LPLD in the MCV.

Conclusions HPSD is an effective alternative strategy for ablation in coronary sinus according to clinical applications and preliminary animal study. However, the safety needs to be further evaluated based on more animal and clinical studies.
\end{abstract}

Keywords High-power and short-duration $\cdot$ Radiofrequency ablation $\cdot$ Coronary sinus $\cdot$ Atrial fibrillation

\section{Introduction}

Coronary sinus-related arrhythmias are common; however, it is difficult to perform radiofrequency (RF) ablation at these sites efficiently and safely due to their anatomical and electrophysiological characteristics. High-power, short-duration ablation (HPSD) was originally used for pulmonary vein isolation (PVI); it improves the relationship between resistive heating and conductive heating and has been proven to be an alternative strategy. Whether it can be applied to ablation in the coronary sinus is unknown. Recently, we have

Xiaomeng Yin

yin_xiaomeng@yeah.net

1 Department of Cardiology, Institute of Cardiovascular Diseases, First Affiliated Hospital of Dalian Medical University, 193\# Lianhe Road, Shahekou District, Dalian 116011, China

2 Department of Graduate school, Dalian Medical University, Dalian, China performed HPSD in the coronary sinus successfully in 4 patients who had unsuccessful treatment with standard power. Then, we investigated the effect of HPSD on lesion creation in the coronary sinus of a swine model.

\section{Methods}

The inspiration for this study design was derived from 4 patients with coronary sinus-related arrhythmias who had unsuccessful ablation with standard power initially, but ultimately received successful ablation under HPSD in our center. The patients provided informed consent for the publication of their cases. The 2 strategies, LPLD followed by HPSD, for each patient were performed by the same operator.

The experimental study showed relevant data to compare the geometric parameters of HPSD lesions and conventional low-power, long-duration ablation (LPLD) lesions in different coronary sinus locations (middle cardiac vein (MCV) and coronary sinus ostium (CSO)). The Institutional Ethical 
Committee for animal research approved the experimental protocol.

\subsection{Case reports}

\subsubsection{Case 1}

A 25-year-old female patient with recurrent palpitations for almost 3 months was diagnosed with atrial tachycardia (AT) in our center. She had no history of structural heart disease. The electrocardiogram (ECG) in the emergency room showed tachycardia with narrow QRS complexes, and her heart rate was 241 beats per minute (Fig. 1a). The RR intervals were consistent, and a $\mathrm{P}$ wave that was negative in the II, III, and aVF leads and positive in the aVL, aVR, and V1 leads was observed at the end of each QRS complex. Intravenous injection of verapamil did not resolve it. Transthoracic echocardiography found a left ventricular ejection fraction (LVEF) of $35 \%$.

A narrow QRS tachycardia with a cycle length (CL) of $302 \mathrm{~ms}$ was induced with programmed stimulation in the atrium during an electrophysiological study (EPS), and it was not influenced by premature ventricular excitation (Fig. 1b). Furthermore, Wenckebach's phenomenon of conduction between the atrium and ventricle was detected for some time, confirming this tachycardia as AT (Fig. 1c). The earliest atrial potential (AP) was observed at $\mathrm{CS} 7,8$ and $50 \mathrm{~ms}$ preceding the onset of the P wave on surface ECG. We placed an irrigatedtip radiofrequency catheter (Thermocool SMART-TOUCH CF-sensing ablation catheter, Biosense Webster, Diamond Bar, Calif) via the femoral vein approach, and a combination of atrial and ventricular potential (VP) and earliest activation was mapped in the MCV (Fig. 1d). Coronary angiography and venography were performed to locate the MCV and assure a safe proximity between it and the adjacent coronary artery (Fig. 1e). RF energy was applied at a maximum power of $20 \mathrm{~W}$ with an irrigation rate of $60 \mathrm{~mL} / \mathrm{min}$ and temperature of $50{ }^{\circ} \mathrm{C}$, and the tachycardia disappeared after $2 \mathrm{~s}$ of application. The application was discontinued once a rise in impedance was noted, and the total application time was $120 \mathrm{~s}$. No tachycardia could be induced by programmed atrial stimulation or following an intravenous drip of isoprenaline. Then, the procedure ended.

Unfortunately, she suffered from AT again 5 hours postprocedure, and the ECG demonstrated the same manifestation as that present pre-procedure. The acute recurrence might be due to inadequate energy delivery in the vessels, and an EPS was carried out again the following day. As suggested by the previous and second EPSs, a satisfactory ablation target was mapped at the same MCV site as in the previous procedure (Fig. 1f, g). We tried the HPSD strategy $(45 \mathrm{~W} / 5 \mathrm{~S})$ with an irrigation rate of $30 \mathrm{~mL} / \mathrm{min}$ and a 56-hole irrigated-tip $\mathrm{RF}$ catheter (Thermocool Smarttouch SF; Biosense Webster,
Diamond Bar, Calif), and the tachycardia disappeared once energy was applied. The tachycardia terminated successfully after a total of 60-s application. No chest pain occurred during energy delivery, and no coronary artery was damaged according to coronary angiography. No tachycardia occurred during the 6-month follow-up, and transthoracic echocardiography demonstrated that the LVEF recovered normally.

\subsubsection{Case 2}

A 28-year-old female patient suffered from palpitations for almost 10 years, and her symptoms worsened within 3 months. The ECG in sinus rhythm demonstrated preexcitation syndrome located in the posterior septum pathway, and tachycardia with a narrow QRS complex is shown in Fig. 2a. The QRS complex in leads II, III, and aVF manifested a "QS" pattern with a negative $\delta$ wave, and that in lead V1 manifested a "rS" pattern with a positive $\delta$ wave. The tachycardia could be terminated by administration of amiodarone or verapamil. She was diagnosed with Wolff-Parkinson-White (WPW) syndrome and admitted to our center. A normal LVEF was found in transthoracic echocardiography, and no structural heart disease was observed.

During an EPS, when pacing at the right ventricular apex, the ventricle-atrium (VA) 1:1 retrograde conduction was centripetal, and the earliest AP was detected in the proximal CS at CS7,8. Decremental VA conduction was not observed. While the CL of atrial S1S1 and S1S2 programmed stimulation decreased, the pre-excitation of the ventricle increased. Atrial stimulation with a CL of $260 \mathrm{~ms}$ induced tachycardia with a narrow QRS complex, which was consistent with clinical ECG. The earliest AP was observed at CS7,8 during tachycardia (Fig. 2b) and orthodromic atrioventricular reentrant tachycardia due to the posterior accessory pathway was confirmed. Mapping was performed on the mitral annulus via atrial septal puncture, and the left accessory pathway was excluded. The RF catheter (Thermocool SMART-TOUCH CFsensing ablation catheter, Biosense Webster, Diamond Bar, Calif) was inserted into the coronary sinus, and a combined atrial-Ventricular $(\mathrm{a}-\mathrm{V})$ electrogram was obtained in $\mathrm{MCV}$ (Fig. 2c, d). The ventricular potential was $30 \mathrm{~ms}$ preceding the onset of the $\delta$ wave on ECG. RF ablation was performed with a setting of $35 \mathrm{~W} / 50^{\circ} \mathrm{C}$, but VA retrograde conduction could not be blocked. Then we tried HPSD strategy. A 56hole irrigated-tip RF catheter (Thermocool Smarttouch SF; Biosense Webster, Diamond Bar, Calif) was positioned at the same site in MCV and VA retrograde conduction was easily blocked within $2 \mathrm{~s}$ following ablation at this site (Fig. 2e). RF energy was applied under the following: $45 \mathrm{~W} / 5 \mathrm{~S}$ with an irrigation rate of $30 \mathrm{~mL} / \mathrm{min}$ and a total RF time of 50 s. After that, ventricular pacing showed VA retrograde conduction disconnected (Fig. 2f), and no tachycardia could be induced by programmed stimulation or an intravenous drip of 
a

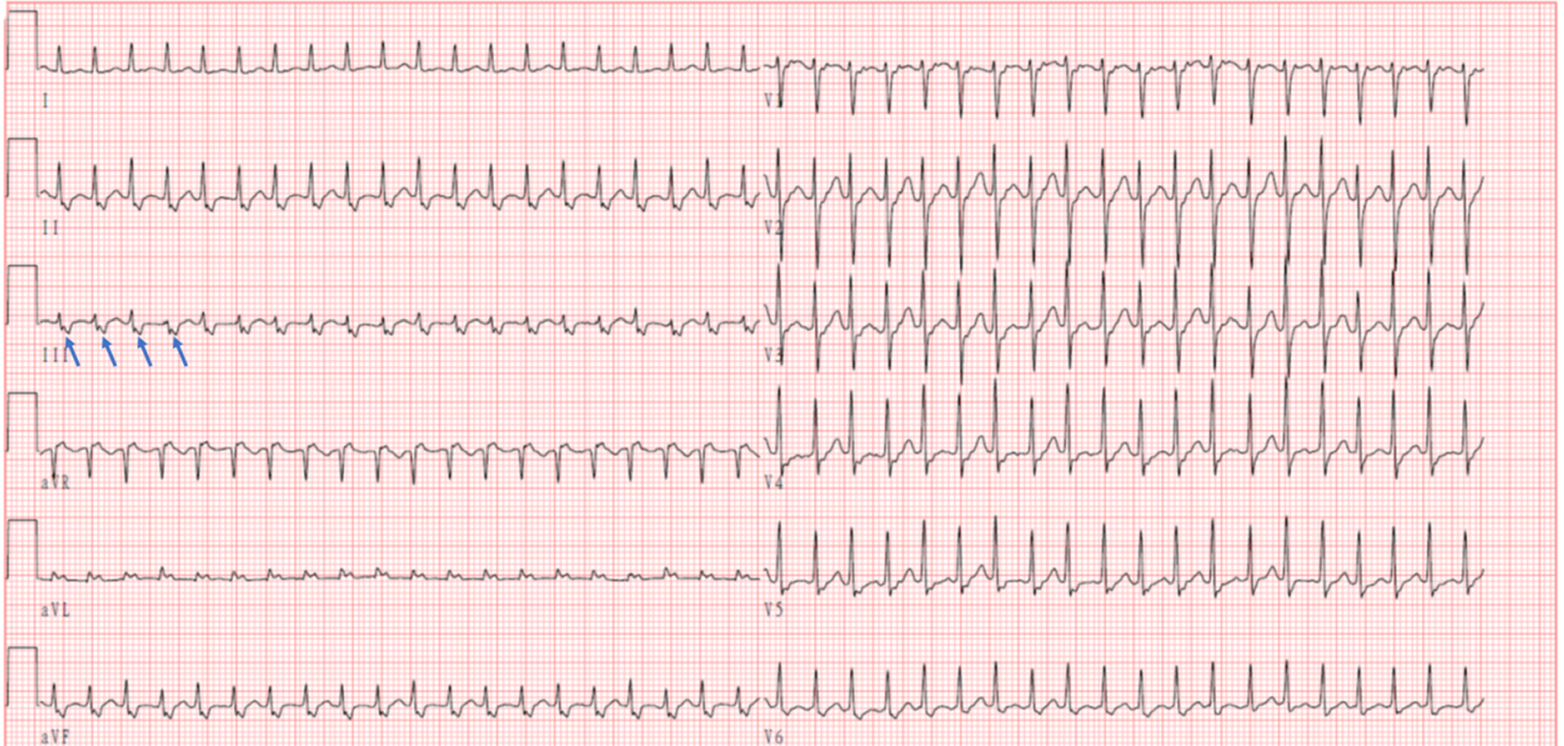

b

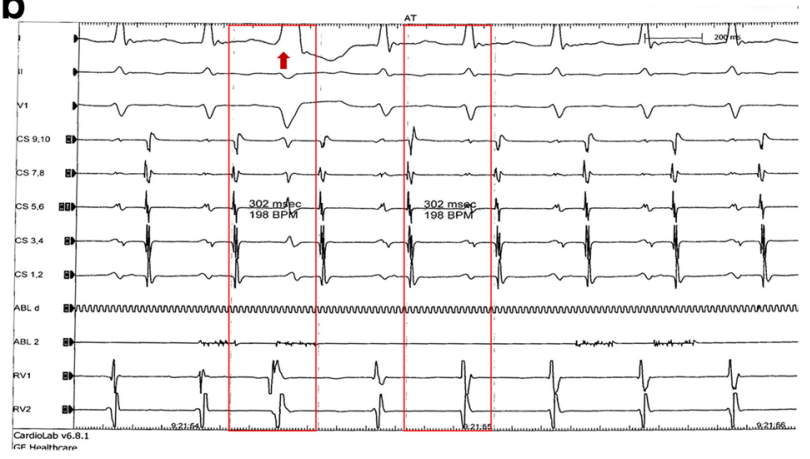

d
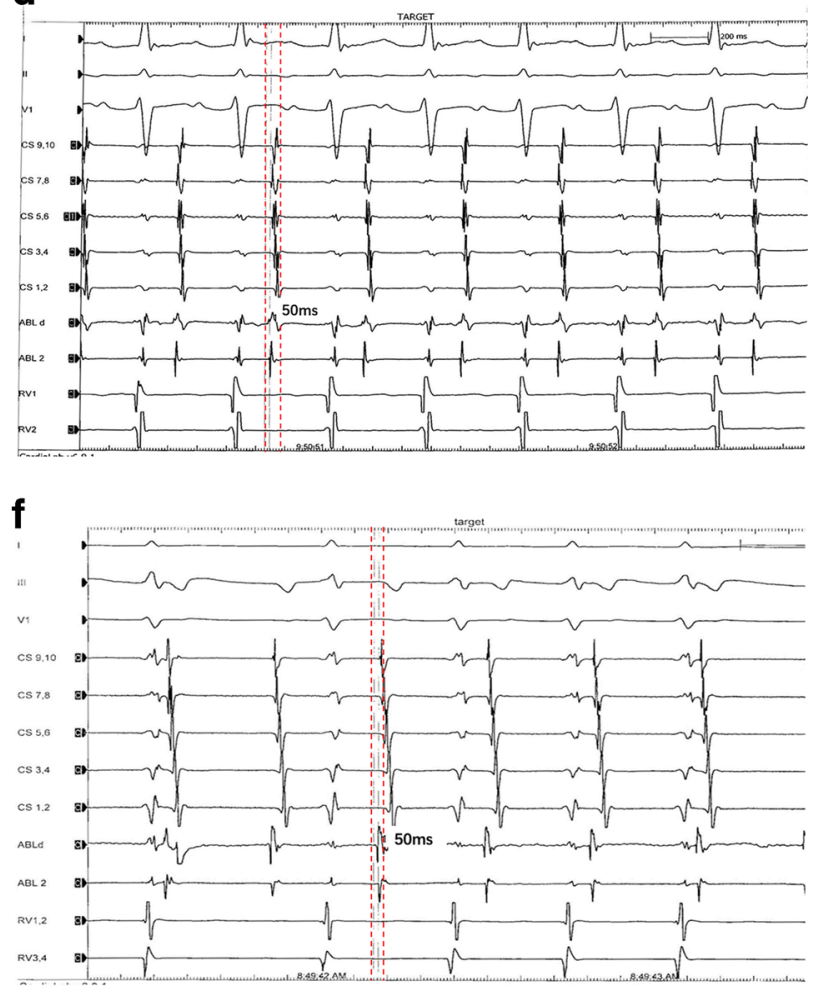

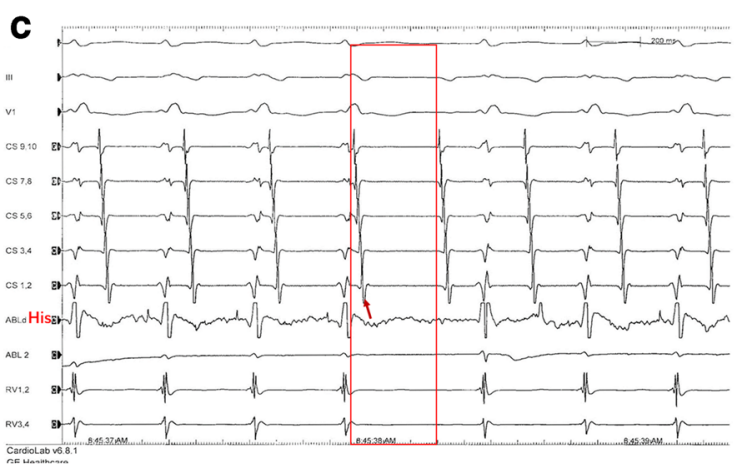

e
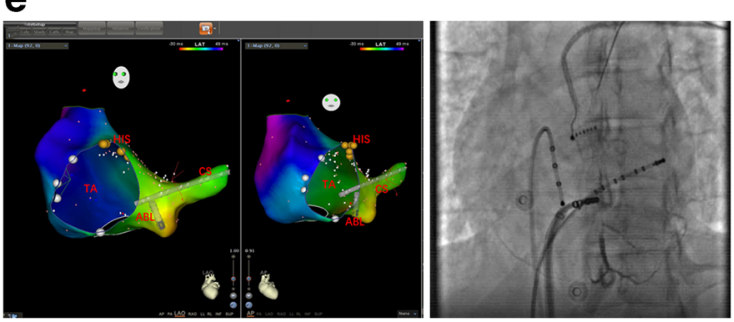

g

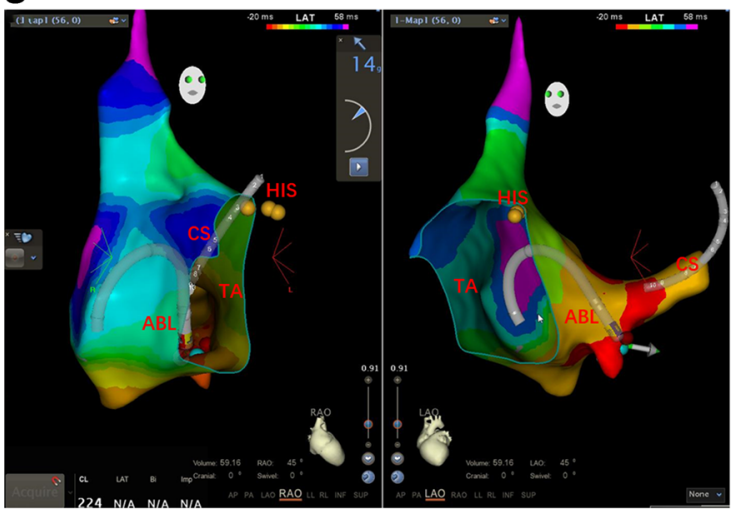


Fig. 1 Case 1-Surface ECG and intracardiac electrogram during EPS study. a A supraventricular tachycardia, P wave (blue arrow) can be detected on end of each narrow QRS complex. b A premature ventricular excitation (red arrow) did not change tachycardia. c, Wenckebach's conduction was founded during tachycardia. Note, the fourth A wave did not conduct to ventricle. d Shows the ablation site. e Left panel shows LAO and anteroposterior view in 3D electroanatomic mapping system (Carto3, Biosense Webster, Diamond Bar, Calif) of ablation site in MCV. Right panel shows coronary venography. f,g, In second procedure, ABL catheter was placed in MCV and obtained satisfactory ablation target which was same as first procedure. (His, the site of His potential; CS, coronary sinus catheter; ABL, ablation catheter; TA, tricuspid annulus)

isoprenaline. No tachycardia occurred during the 12-month follow-up, and no ventricular pre-excitation was detected in sinus rhythm.

\subsubsection{Case 3}

A 66-year-old female patient with frequent premature ventricular contractions (PVCs) came to our center with a history of palpitations for 1 year. She took $\beta$-blockers and propafenone, which had poor effects. She had no structural heart disease, coronary heart disease, or hypertension. The ECG and ambulatory ECG demonstrated monomorphic PVCs with the morphology of a right bundle branch block (RBBB) in the V1 lead, a positive QRS complex in the II, III, and aVF leads, and a "QS" pattern in the I lead, suggesting the origin of the left ventricular outflow tract (LVOT) (Fig. 3a). During the EPS, the earliest ventricular potential (VP), which was $27 \mathrm{~ms}$ preceding the onset of the QRS wave on the surface ECG, was detected in the left coronary cusp. RF ablation was performed via an ablation catheter (Thermocool SMARTTOUCH CF-sensing ablation catheter, Biosense Webster, Diamond Bar, Calif) with a setting of $35 \mathrm{~W} / 50^{\circ} \mathrm{C}$, and the PVCs decreased but did not disappear. Then, we moved the ablation catheter forward to the mitral annulus, but we obtained no earlier VP. The PVCs did not vanish when RF ablation was performed with a same setting at the high septum of the right ventricular outflow tract (RVOT), where the VP was $21 \mathrm{~ms}$ preceding the onset of the surface QRS wave. Then, we placed an RF catheter distal to the GCV and obtained a VP that was $21 \mathrm{~ms}$ preceding the onset of the QRS wave on the surface ECG (Fig. 3b, c). Coronary angiography and venography were performed to identify the GCV and ensure a safe proximity between it and the adjacent coronary artery. RF energy was applied in the GCV with a 56-hole irrigated-tip radiofrequency catheter (Thermocool Smarttouch SF; Biosense Webster, Diamond Bar, Calif) at a maximum power of $45 \mathrm{~W}$, an irrigation rate of $30 \mathrm{~mL} / \mathrm{min}$ and a temperature of $50^{\circ} \mathrm{C}$ for $5 \mathrm{~s}$, and the PVCs vanished immediately. The total application time was $25 \mathrm{~s}$, and no PVC occurred during the following $30 \mathrm{~min}$. No coronary artery damage was confirmed after coronary angiography. No tachycardia occurred at the 15-month follow-up.

\subsubsection{Case 4}

A 49-year-old man with frequent PVCs suffered from palpitations and shortness of breath for 2 months. He had no structural heart disease, coronary heart disease, or hypertension. The echocardiography was normal. The ambulatory ECG demonstrated monomorphic PVCs and non-sustained ventricular tachycardia (NSVT). The surface ECG showed PVCs with the morphology of a left bundle branch block (LBBB), a positive QRS complex in the II, III, and aVF leads, and an "rS" pattern in the I lead and late transition in the precordial lead, suggesting the origin of the RVOT (Fig. 4a). During the EPS, we mapped the earliest VP at the high septum of the RVOT, which was $31 \mathrm{~ms}$ preceding the onset of the QRS wave on the surface ECG. RF energy was applied at 35 $\mathrm{W} / 50^{\circ} \mathrm{C}$ with a catheter (Thermocool SMART-TOUCH CFsensing ablation catheter, Biosense Webster, Diamond Bar, Calif), and PVCs became less frequent but did not disappear. Then, we obtained a VP distal to the GCV, which was $27 \mathrm{~ms}$ preceding the onset of the QRS wave on the surface ECG (Fig. $4 \mathrm{~b}, \mathrm{c})$. RF energy was administered at the site by a 56-hole irrigated-tip RF catheter (Thermocool Smarttouch SF; Biosense Webster, Diamond Bar, Calif), after confirming safe proximity, with a maximum power of $45 \mathrm{~W}$, an irrigation rate of $30 \mathrm{~mL} / \mathrm{min}$ and a temperature of $50{ }^{\circ} \mathrm{C}$, and the PVCs vanished immediately. The total application time was $25 \mathrm{~s}$, and no PVC occurred during the following $30 \mathrm{~min}$. No coronary artery damage was detected on coronary angiography. No PVC or VT occurred at the 5-month follow-up.

\subsection{Animal study}

\subsubsection{Swine heart preparation}

Four swine hearts (all males, weight $450 \mathrm{~g} \pm 15 \mathrm{~g}$, age 12-15 months) were prepared in vitro. The isolated heart model was based on the method described by Langendorff [1]. It consisted of a saline-filled container, including a thermostat to keep the temperature at $37^{\circ} \mathrm{C}$, a circulating pump, an indifferent electrode, and porcine heart preparations immersed in saline after pericardium excision. The heart was filled with heparinized swine blood with a fluid temperature of $37^{\circ} \mathrm{C}$ at a perfusion pressure of $60-70 \mathrm{mmHg}$ and flow velocity of 160 $\mathrm{mL} / \mathrm{min}$ in the coronary sinus to simulate the characteristics of blood flow in vivo [2].

\subsubsection{Ablation protocol}

The procedure was carried out at the CSO and MCV. The initial impedance was 190-210 $\Omega$, and the force range was 
a

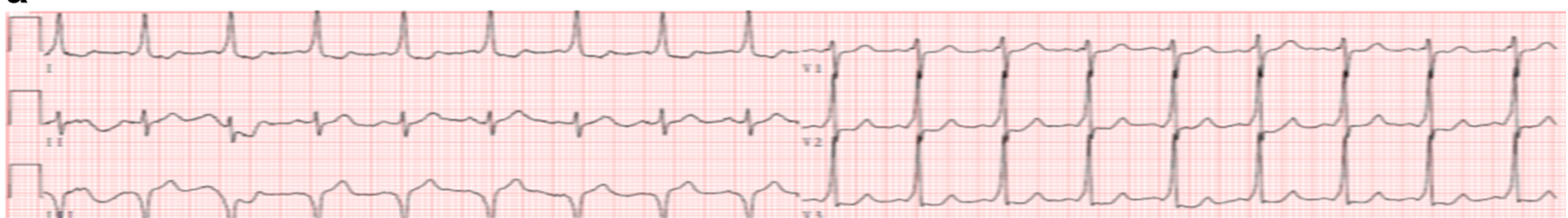

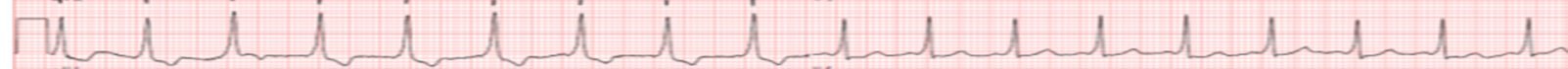

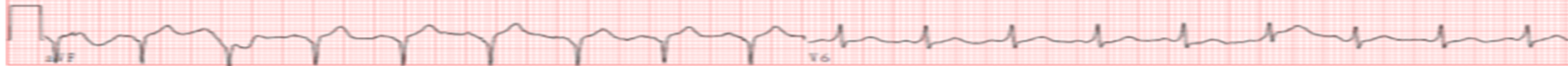

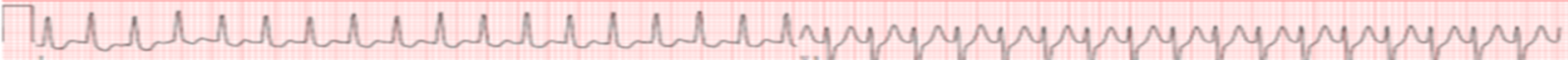

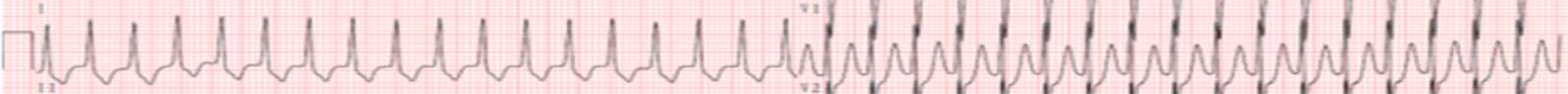

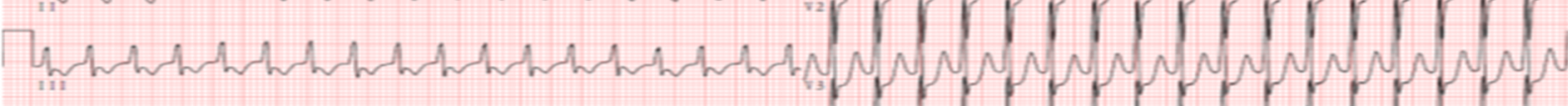

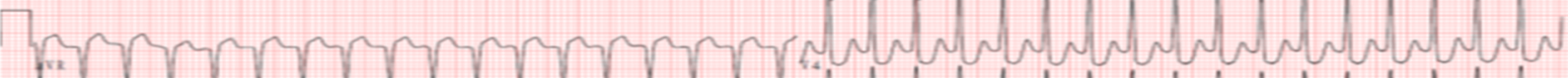

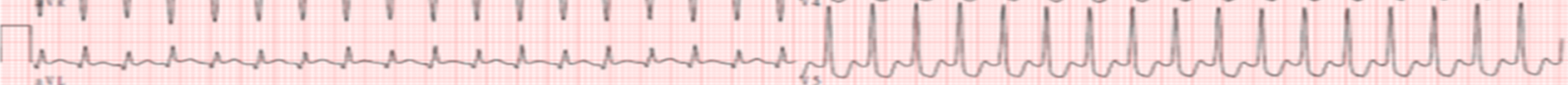

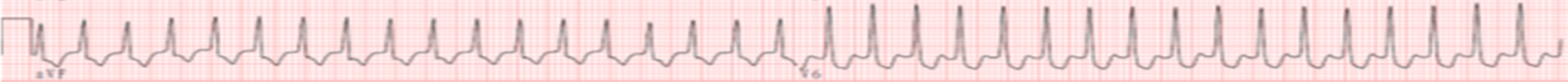

b

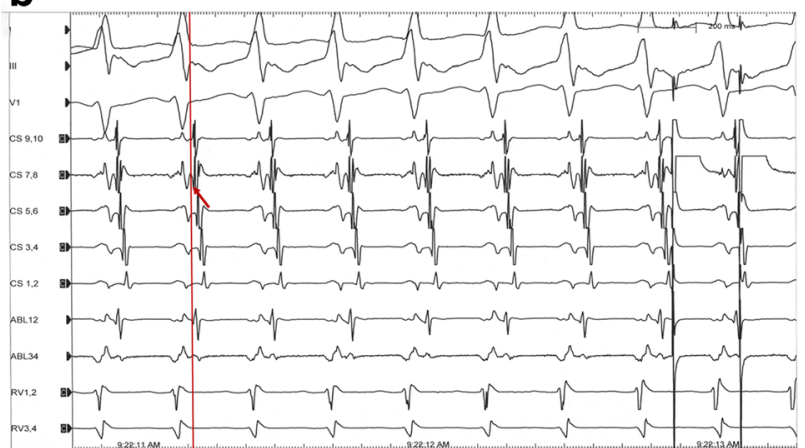

d

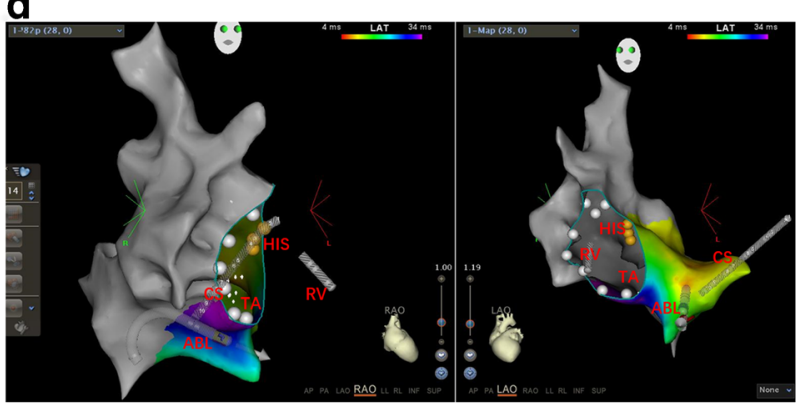

C

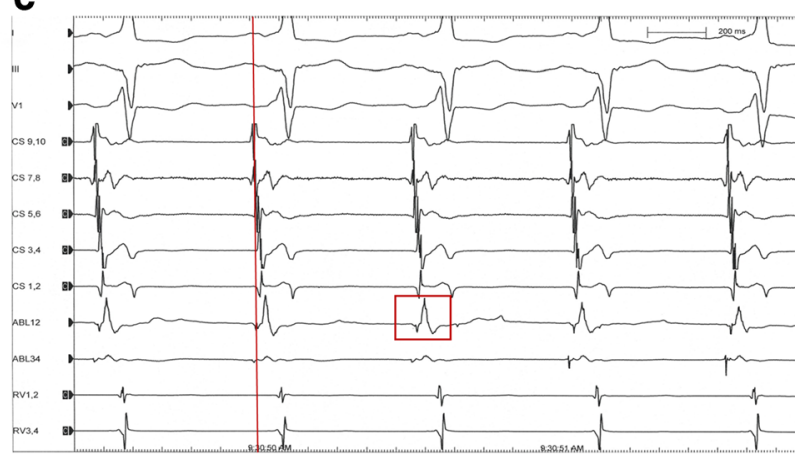

e

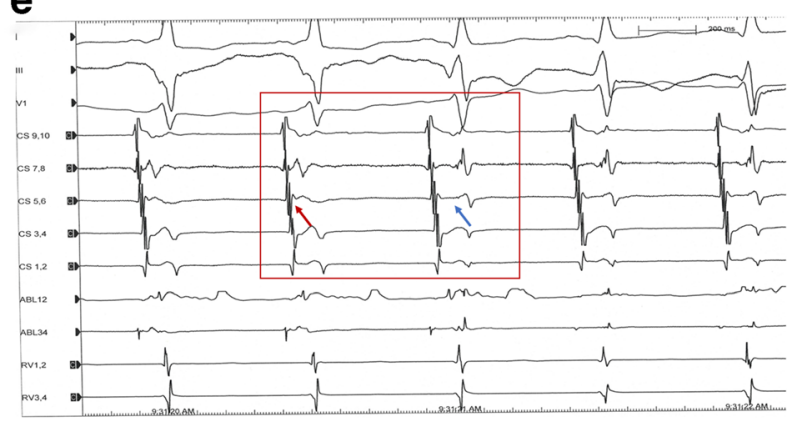

f

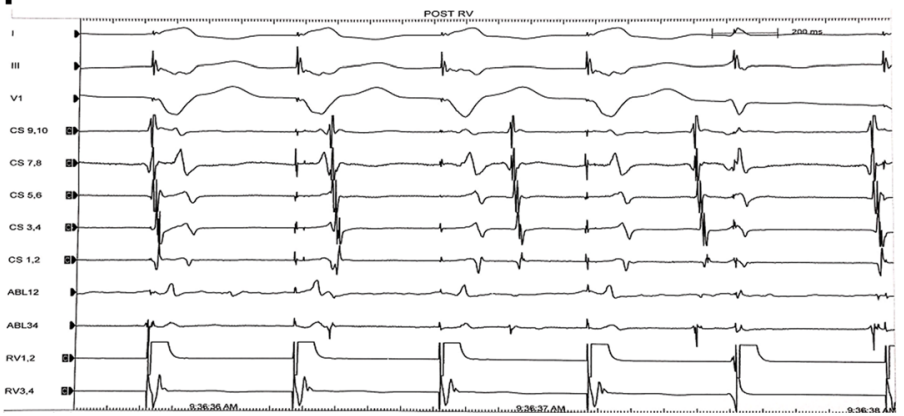


Fig. 2 Case 2-Surface ECG and intracardiac electrogram during EPS study. a The upper figure shows pre-excitation of ventricle in sinus rhythm. The below figure demonstrates the tachycardia. b The earliest retrograde $\mathrm{AP}$ was detected in CS7,8 during tachycardia. $\mathbf{c} \mathrm{ABL}$ catheter recorded the earliest antegrade ventricular activation (vertical red line) in sinus rhythm and a combined a-V electrogram (red box). d Shows the ablation site. e Ablation at MCV terminates pre-excitation of ventricle successfully (red box). The fusion of AV potential (red arrow) dissociated (blue arrow) during ablation. f VA retrograde conduction was terminated

10-20 g. The titration RF ablation was performed under $45 \mathrm{~W} /$ $5 \mathrm{~S}, 5$ rounds, for a total duration of $25 \mathrm{~s}$, in the study group (HPSD: $45 \mathrm{~W} / 5 \times 5 \mathrm{~S}$ ). In the control group, titration ablation was performed under $25 \mathrm{~W} / 10 \mathrm{~S}, 5$ rounds, for a total duration of $50 \mathrm{~s}$ (LPLD: $25 \mathrm{~W} / 5 \times 10 \mathrm{~S}$ ). RF ablation was performed with a 56-hole irrigated-tip RF catheter (Thermocool Smarttouch SF; Biosense Webster, Diamond Bar, Calif). The saline irrigation rate for the catheter tip was set at 30
$\mathrm{mL} / \mathrm{min}$ for HPSD and at $25 \mathrm{~mL} / \mathrm{min}$ for LPLD. The saline irrigation rate was maintained at $2 \mathrm{~mL} / \mathrm{min}$ between applications. The application was terminated immediately if steam pop occurred.

\subsubsection{Lesion examination}

Lesion length was defined as the maximal longitudinal surface diameter, and lesion width was defined as the maximal horizontal surface diameter. The heart was cut along the maximal longitudinal surface diameter to measure lesion depth, and the following parameters were determined: maximal depth (D), maximal width (W), and maximal length (L). Because the lesions were made with the catheter in a position parallel to the tissue, the lesion volume was calculated with the formula for an ellipsoid as follows [3]:

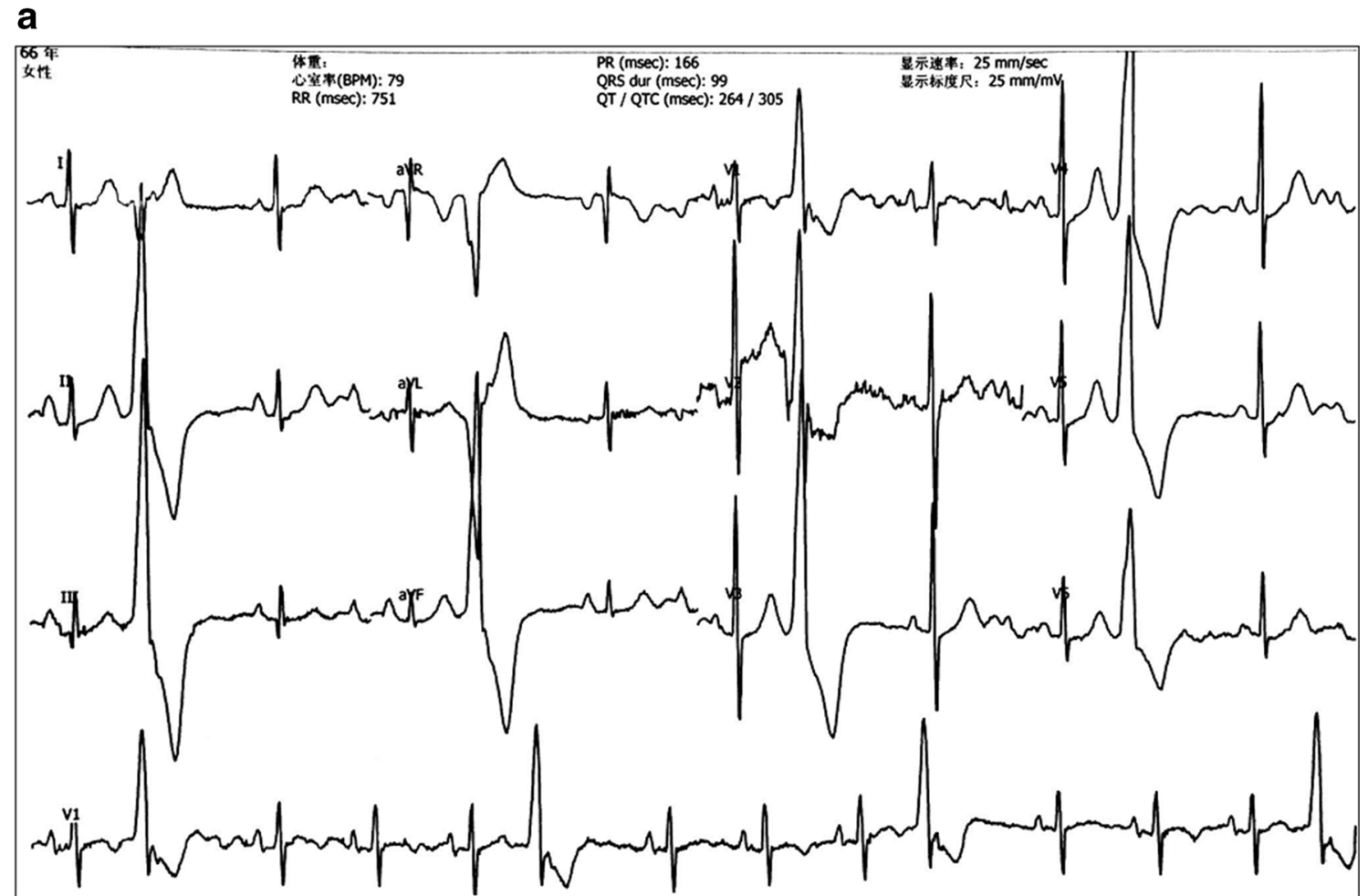

b

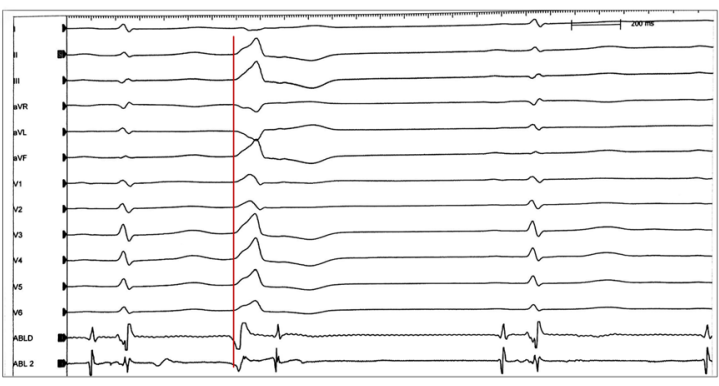

C

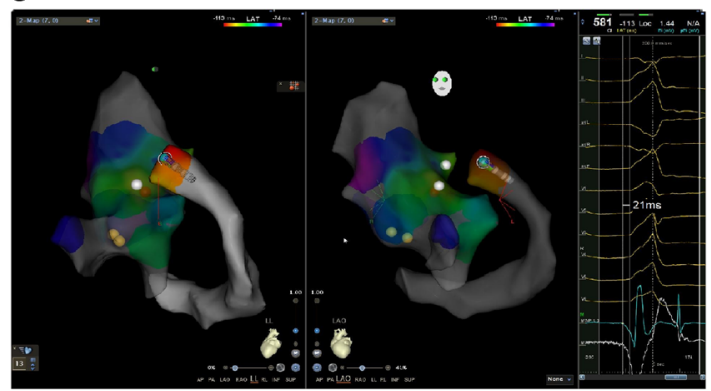

Fig. 3 Case 3-surface ECG and intracardiac electrogram during EPS study. a The ECG demonstrated PVC. b, c Shows the ablation site 
a

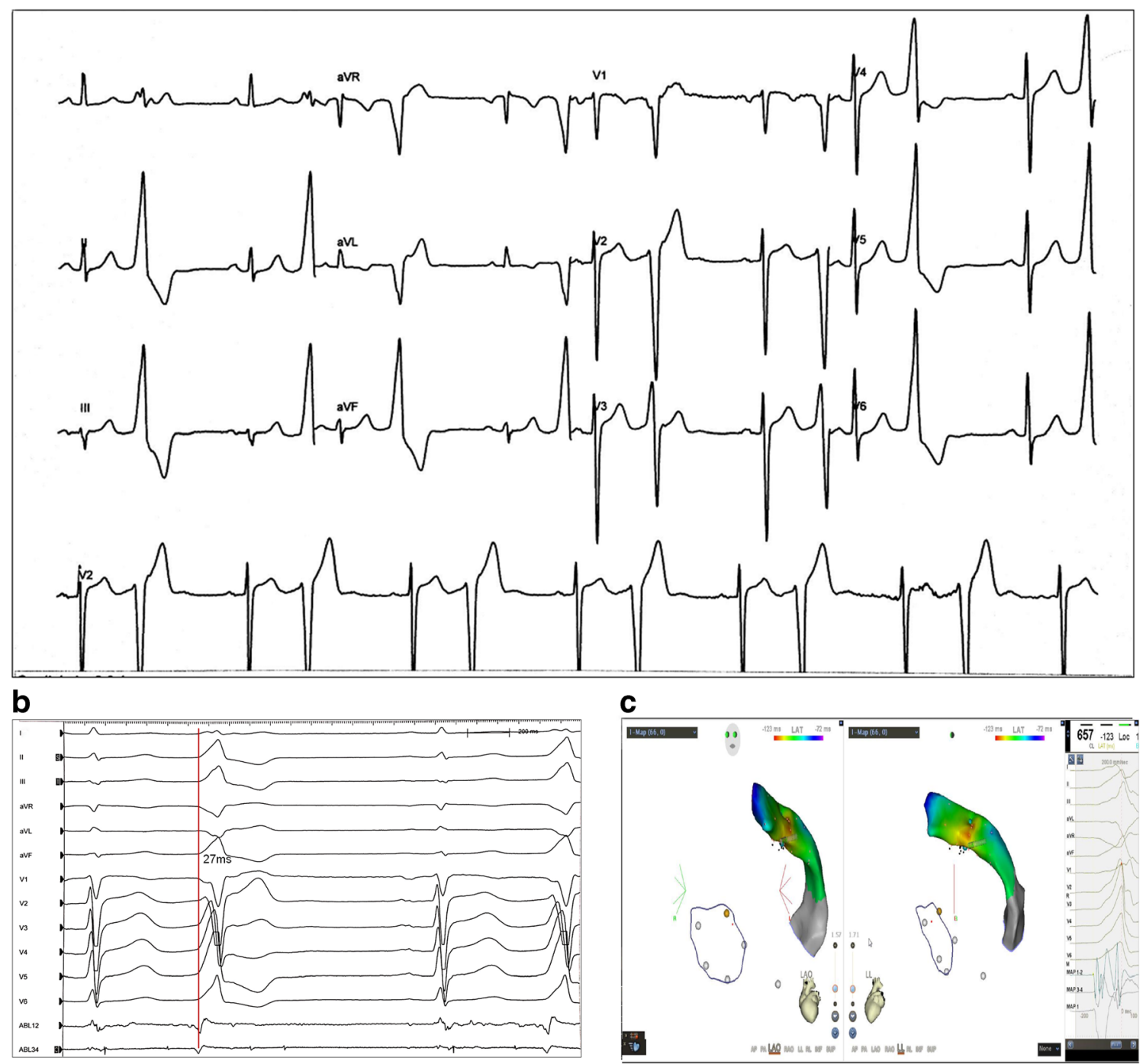

Fig. 4 Case 4 - surface ECG and intracardiac electrogram during EPS study. a The ECG demonstrated PVC. b, c Shows the ablation site

Lesion Volume $=\left(\frac{4}{3} \pi \times D \times \frac{1}{2} W \times \frac{1}{2} L\right) \times \frac{1}{2}$.

\subsubsection{Statistical analysis}

All analyses were performed using SPSS version 22.0 (SPSS Inc., Chicago, IL, USA). Continuous variables are expressed as the mean \pm SD if normally distributed; the median and the 25 to $75 \%$ interquartile range were used if the data were clearly skewed. An unpaired $t$ test or one-way analysis of variance was performed for measurement data. For categorical variables, chi-square tests or Fisher's exact tests were used. A 2-tailed $P$ value < 0.05 was considered statistically significant.

\section{Results}

\subsection{RF ablation with HPSD and LPLD}

There were 28 lesions created in a total of 4 swine hearts, including 12 ablations produced in the $\mathrm{CSO}$ and 16 produced in the MCV (Fig. 5). HPSD lesions ( $45 \mathrm{~W} / 25 \mathrm{~S}, n=16)$ and LPLD lesions $(25 \mathrm{~W} / 50 \mathrm{~S}, n=12)$ are compared in Table 1. HPSD lesions were longer, wider, deeper, and larger than LPLD lesions. However, there was a significant difference only in mean width $(9.34 \pm 2.14 \mathrm{~mm}$ vs. $7.78 \pm 1.71 \mathrm{~mm}$, $P=0.041$ ) between the 2 groups.

\subsection{RF ablation at the CSO}

A total of 12 ablations were carried out in the $\mathrm{CSO}, 4$ of which used the LPLD strategy, while the other 8 used the HPSD 
Fig. 5 Gross appearance of swine heart tissue. (HPSD, high-power, short-duration; LPLD, low-power, long-duration; CSO, coronary sinus ostium; MCV, middle cardiac vein)
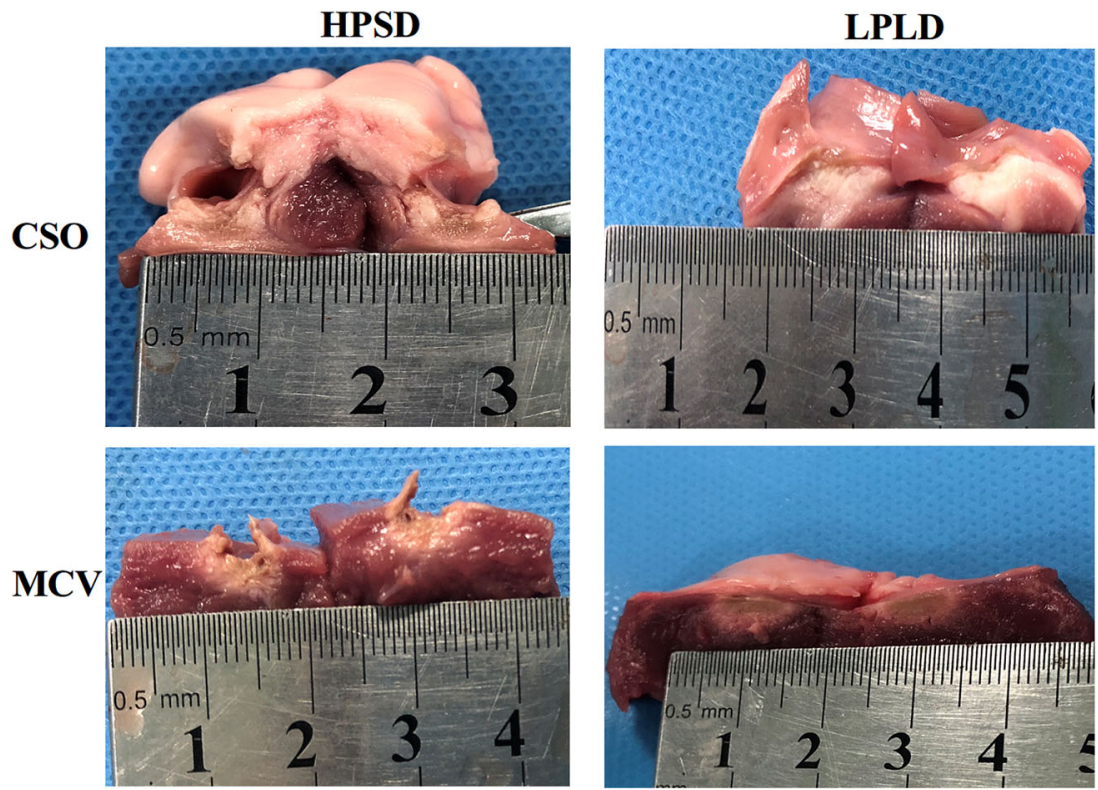

strategy. Lesion metrics are shown in Table 2, and no significant differences were found in geometric parameters between the 2 groups.

\subsection{RF ablation at the MCV}

A total of 16 ablations were carried out in the MCV, 4 of which were produced under the LPLD strategy, and another 8 were produced under the HPSD strategy. Lesion metrics are shown in Table 2. In brief, HPSD produced significantly deeper (4.68 $\pm 0.75 \mathrm{~mm}$ vs. $3.299 \pm 0.42 \mathrm{~mm}, P=0.026)$ and larger $(393.35 \pm 129.48 \mathrm{~mm} 3$ vs. $253.29 \pm 84.9 \mathrm{~mm}$, $P=0.023$ ) lesions than LPLD. One instance of steam pop occurred in the LPLD group.

\section{Discussion}

In this study, we presented clinical application of HPSD in 4 patients with 3 different types of arrhythmias and observed the geometric characteristics of lesions created by HPSD in the coronary sinus in swine hearts. The main finds of this study show that HPSD produce longer, wider, deeper, and larger lesions than LPLD in coronary sinus; moreover, this difference was more pronounced when the ablation was in the MCV. HPSD has a reliable efficiency not only for PVI but also for ablation in the coronary sinus.

\subsection{HPSD}

RF ablation lesions are generated by thermal injury: resistive heating and conductive heating. Resistive heating occurs on tissues immediately, and conductive heating occurs slowly but contributes more to lesion growth. Conductive heating may extend to deeper tissue layers and result in peripheral tissue damage. HPSD improves the relationship between resistive heating and conductive heating, allowing immediate heating to result in a larger, permanent tissue injury while restricting conductive heating. Felix Bourier et al. [4] compared lesion metrics of HPSD application and standard power application in an in silico simulation and a porcine in vitro model. HPSD can produce a larger and shallower lesion, and lesion metrics increase fastest during the first $10 \mathrm{~s}$ regardless of the power setting. Eran Leshem et al. [5] researched the characteristics of HPSD lesions in 20 beating swine hearts and found that HPSD produce a wider, transmural lesion with $100 \%$ contiguous lines, compared with standard power ablation, which yields smaller lesions with $25 \%$ linear gaps and $29 \%$ partial
Table 1 Geometrical characteristics of HPSD lesions and LPLD lesions

\begin{tabular}{lccc}
\hline & HPSD lesions (45 W/25 S, $n=16)$ & LPLD lesions (25 W/50 S, =12) & $P$-value \\
\hline Lesion length, mm & $8.6 \pm 1.4$ & $8.43 \pm 1.61$ & 0.765 \\
Lesion width, mm & $9.34 \pm 2.14$ & $7.78 \pm 1.71$ & 0.041 \\
Lesion depth, mm & $4.66 \pm 1.12$ & $4.29 \pm 0.99$ & 0.375 \\
Lesion volume, $\mathrm{mm}^{3}$ & $410.95 \pm 169.58$ & $310.5 \pm 196.52$ & 0.159 \\
\hline
\end{tabular}

HPSD high-power, short-duration; LPLD low-power, long-duration 
Table 2 Geometrical characteristics of HPSD lesions and LPLD lesions at CSO and $\mathrm{MCV}$

\begin{tabular}{lllr}
\hline & HPSD lesions (45 W/25 S) & LPLD lesions (25 W/50 S) & $P$-value \\
\hline \multicolumn{1}{c}{ CSO, $n$} & 8 & 4 & 12 \\
Lesion length, mm & $8.72 \pm 1.76$ & $8.36 \pm 1.87$ & 0.748 \\
Lesion width, mm & $9.44 \pm 2.21$ & $8.66 \pm 1.39$ & 0.533 \\
Lesion depth, mm & $4.64 \pm 1.48$ & $5.03 \pm 1.43$ & 0.67 \\
Lesion volume, $\mathrm{mm}^{3}$ & $428.54 \pm 210.11$ & $424.93 \pm 314$ & 0.981 \\
Steam pop, $\mathrm{n}$ & 0 & 0 & 0 \\
$\quad M C V, n$ & 8 & 8 & 16 \\
Lesion length, mm & $8.47 \pm 1.02$ & $8.46 \pm 1.6$ & 0.985 \\
Lesion width, mm & $9.38 \pm 2.23$ & $7.34 \pm 1.76$ & 0.065 \\
Lesion depth, mm & $4.68 \pm 0.75$ & $3.299 \pm 0.42$ & 0.026 \\
Lesion volume, mm ${ }^{3}$ & $393.35 \pm 129.48$ & $253.29 \pm 84.9$ & 0.023 \\
Steam pop, $\mathrm{n}$ & 0 & 1 & 1 \\
\hline
\end{tabular}

HPSD high-power, short-duration; $L P L D$ low-power, long-duration; $C S O$ coronary sinus ostium; $M C V$ middle cardiac vein thickness. Our study compared the differences in geometric parameters of lesions between HPSD and LPLD in the coronary sinus. The HPSD lesions were larger and deeper than LPLD lesions, but the difference was significant only in the $\mathrm{MCV}$. It may be due to the low blood flow in the coronary sinus and its different anatomical structures from the pulmonary vein.

Nilsson B et al. [6] compared the effectiveness of segmental PVI between HPSD and traditional application. HPSD reduced PVI time, mean fluoroscopy time with a similar outcome. The efficacy and safety of very high-power, shortduration ablation was also evaluated [7]. The procedure and fluoroscopy times were lower than those of conventional ablation, with no increased complications.

Castrejón-Castrejón et al. [8] found that asymptomatic esophageal lesions occur more frequently in conventional applications than in HPSD according to upper digestive tract endoscopy. The authors indicated that longer application time play more important role in esophageal damage than the power setting. HPSD did not increase the risk of esophageal thermal injury [9]. Winkle RA et al. [10] examined complication rates for 13974 of ablations at RF powers ranging from 45-50 $\mathrm{W}$ in 10,284 patients from 4 centers. Three atrial esophageal fistulas occurred in the lower-power group $(35 \mathrm{~W} / 20 \mathrm{~s}$ on the posterior wall), but only 1 atrial esophageal fistula occurred in the high-power group (45-50 W/2-10 s on the posterior wall). The incidence of audible steam pop, pericardial effusion, and esophageal lesions was extremely low in the HPSD group.

Although HPSD shows a comparable safety profile to conventional ablation, the safety margin of HPSD is very narrow. To predict lesion dimensions properly, some algorithms were created and have been proven to be effective and safe $[11,12]$. It was shown that high-power ablation guided by the ablation index (AI) is safe and efficient [13, 14]. However, a variety of
AI or lesion size index (LSI) values were set in these studies, and the explicit values are still in question, especially when the posterior wall is ablated. Unipolar signal modification may be another effective parameter for guiding high-power RF ablation for PVI [15]. However, it is only used in ablation for paroxysmal $\mathrm{AF}$, and it cannot distinguish electrical stunning from cellular necrosis, which may result in false-positive transmural lesions, especially in conventional power ablation.

The acute outcome of HPSD has been proven, but its longterm outcome is still controversial [16]. Moreover, the specific reproducible ablation parameters and endpoint should be determined, and whether the power be reduced when ablating posterior wall is still a question.

\subsection{Electrophysiological characteristics of the coronary sinus}

The coronary sinus is in the atrioventricular groove, and it is surrounded by myocardial sleeves, which are composed of bands of muscle from the left atrium (LA) and right atrium (RA), some of which have ectopic automaticity [17]. It was named the coronary sinus muscle extension (CSE), and these specific anatomical sites and adjacent pericardial tissues may be sources of arrhythmias such as AT, AVRT, PVC, and idiopathic VT. It is difficult to produce transmural, durable lesions at these sites because of its complex anatomy, the venous characteristics of its large lumen and thin wall, interrupted ablation due to increased impedance, and inadequate power delivery due to the effect of a "heat sink" caused by blood flow in the coronary sinus system [18]. The coronary sinus and its branches are close to the left circumflex artery and posterolateral branches of the right coronary artery; thus, it is critical to avoid damage to the vein and adjacent coronary artery during ablation. The strategy of lower power and a short 
duration and performing angiography before the procedure to determine anatomical proximity are useful to reduce the risk of coronary injuries $[19,20]$. However, low power may lead to insufficient ablation, and a long duration may generate discontinuous energy delivery due to increased impedance, leading to extra damage to collateral tissue. Obtaining sufficient energy delivery and avoiding damage to adjacent tissue are critical to successful ablation in the coronary sinus [21].

\subsection{HPSD in the coronary sinus}

As mentioned above, increasing the power setting properly with a short duration may be an alternative ablation protocol with a promising safety status. HPSD was applied to in GCV (maximum power $50 \mathrm{~W}, 30 \mathrm{~s}$ for each lesion) in a patient with idiopathic VT [22]. No arrhythmia occurred during the 8month follow-up after the procedure.

The feasibility and safety of HPSD in the coronary sinus were also verified in our study. Titrated HPSD can produce lesions that are approximately similar in length, width, depth, and volume in a short time. The volume and depth of titrated HPSD lesions are larger than those of LPLD lesions. It is interesting that the difference is more significant in the $\mathrm{MCV}$, which may be due to the weak effect of the "heat sink" because of poor blood flow in the MCV [18]. No "pop" occurred in HPSD.

We performed HPSD at the MCV, CSO, and GCV after a previous unsuccessful ablation with the traditional LPLD strategy in 4 patients, and a satisfactory outcome was achieved. RF ablation was performed by a 56-hole irrigatedtip RF catheter with an irrigation rate of $30 \mathrm{~mL} / \mathrm{min}$ and a temperature of $50{ }^{\circ} \mathrm{C}$. In consideration of the possible damage to coronary sinus with high power, each HPSD procedure only lasted for $5 \mathrm{~s}(45 \mathrm{~W} / 5 \mathrm{~S})$ and 5 rounds were performed. No coronary artery damage was confirmed according to the coronary angiography.

\subsection{Limitations}

The study has several limitations. First, the animal study was an in vitro experiment not an in vivo experiment, and HPSD was carried out on non-beating hearts. The isolated heart model may not fully reflect real blood flow in the coronary sinus, influencing the experimental results. So, the data are not fully representative of lesions characteristics in patients. Second, the sample size of the animal research and the number of clinical cases are small, and there is a lack of histological observation of HPSD lesions. Four case reports are inadequate to demonstrate safety. More in vivo animal studies and clinical cases are needed. Third, the evaluation of complications and risk was only focused on coronary artery damage; however, we did not assess the risk of other complications, such as esophageal injury. Fourth, none of the 4 patients had structural heart disease or coronary heart disease, and whether HPSD can be applied to these patients remains unclear. Finally, the feasibility of HPSD in other arrhythmias, such as atrial flutter and VT, needs further study.

\section{Conclusion}

Compared with conventional ablation procedures, HPSD in the coronary sinus is efficacious. Although the safety needs to be further evaluated based on more animal and clinical studies, HPSD is an alternative strategy for ablation in the coronary sinus.

Acknowledgements We thank AJE (www.aje.cn) for its linguistic assistance during the preparation of this manuscript.

Author contribution All authors read and approved the final manuscript.

Data availability All the data supporting our findings are contained within the manuscript.

\section{Declarations}

Ethics approval All procedures performed in studies involving human participants were in accordance with the 1964 Declaration of Helsinki and its later amendments or comparable ethical standards.

Consent to participate All participants agreed to participate and signed written consents.

Consent for publication Written informed consents were obtained from all the patients.

Competing interests The authors declare no competing interests.

Open Access This article is licensed under a Creative Commons Attribution 4.0 International License, which permits use, sharing, adaptation, distribution and reproduction in any medium or format, as long as you give appropriate credit to the original author(s) and the source, provide a link to the Creative Commons licence, and indicate if changes were made. The images or other third party material in this article are included in the article's Creative Commons licence, unless indicated otherwise in a credit line to the material. If material is not included in the article's Creative Commons licence and your intended use is not permitted by statutory regulation or exceeds the permitted use, you will need to obtain permission directly from the copyright holder. To view a copy of this licence, visit http://creativecommons.org/licenses/by/4.0/.

\section{References}

1. Bell RM, Mocanu MM, Yellon DM. Retrograde heart perfusion: the Langendorff technique of isolated heart perfusion. J Mol Cell Cardiol. 2011;50(6):940-50. https://doi.org/10.1016/j.yjmcc.2011. 02.018 . 
2. Motayagheni N. Modified Langendorff technique for mouse heart cannulation: improved heart quality and decreased risk of ischemia. MethodsX. 2017;4:508-12. https://doi.org/10.1016/j.mex.2017.11. 004.

3. EVERETT TH IV, LEE KW, WILSON EE, GUERRA JM, VAROSY PD, OLGIN JE. Safety profiles and lesion size of different radiofrequency ablation technologies: a comparison of large tip, open and closed irrigation catheters. J Cardiovasc Electrophysiol. 2009;20(3):325-35. https://doi.org/10.1111/j.1540-8167.2008. 01305.x.

4. Bourier F, Duchateau J, Vlachos K, Lam A, Martin CA, Takigawa $\mathrm{M}$, et al. High-power short-duration versus standard radiofrequency ablation: insights on lesion metrics. J Cardiovasc Electrophysiol. 2018;29(11):1570-5. https://doi.org/10.1111/jce.13724.

5. Leshem E, Zilberman I, Tschabrunn CM, Barkagan M, ContrerasValdes FM, Govari A, et al. High-power and short-duration ablation for pulmonary vein isolation biophysical characterization. Jacc Clin Electrophysiol. 2018;4(4):467-79. https://doi.org/10.1016/j. jacep.2017.11.018.

6. Nilsson B, Chen X, Pehrson S, Svendsen JH. The effectiveness of a high output/short duration radiofrequency current application technique in segmental pulmonary vein isolation for atrial fibrillation. Ep Europace. 2006;8(11):962-5. https://doi.org/10.1093/europace/ eul100.

7. Reddy VY, Grimaldi M, Potter TD, Vijgen JM, Bulava A, Duytschaever MF, et al. Pulmonary vein isolation with very high power-short duration temperature-controlled lesions: the first-inhuman QDOT-FAST multicenter trial. Jacc Clin Electrophysiol. 2019;5:778-86. https://doi.org/10.1016/j.jacep.2019.04.009.

8. Castrejón-Castrejón S, Cossiani MM, Molina MO, Escobar C, Torres CF, Bada NG, et al. Feasibility and safety of pulmonary vein isolation by high-power short-duration radiofrequency application: short-term results of the POWER-FAST PILOT study. J Interventional Cardiac Electrophysiol Int J Arrhythm Pacing. 2019:1-9. https://doi.org/10.1007/s10840-019-00645-5.

9. Baher A, Kheirkhahan M, Rechenmacher SJ, Marashly Q, Kholmovski EG, Siebermair J, et al. High-power radiofrequency catheter ablation of atrial fibrillation. Jacc Clin Electrophysiol. 2018;4(12):1583-94. https://doi.org/10.1016/j.jacep.2018.07.017.

10. Winkle RA, Mohanty S, Patrawala RA, Mead RH, Kong MH, Engel $\mathrm{G}$, et al. Low complication rates using high power (45-50 W) for short duration for atrial fibrillation ablations. Heart Rhythm. 2019;16(2):165-9. https://doi.org/10.1016/j.hrthm.2018.11.031.

11. Seitz J, Bars C, Théodore G, Beurtheret S, Lellouche N, Bremondy $\mathrm{M}$, et al. AF ablation guided by spatiotemporal electrogram dispersion without pulmonary vein isolation. J Am Coll Cardiol. 2017;69(3):303-21. https://doi.org/10.1016/j.jacc.2016.10.065.

12. Koruth JS, Iwasawa J, Enomoto Y, Bar-Tal M, Ultchin Y, Sigal A, et al. Chamber-specific radiofrequency lesion dimension estimation using novel catheter-based tissue interface temperature sensing. Jacc Clin Electrophysiol. 2017;3(10):1092-102. https://doi.org/10. 1016/j.jacep.2017.08.008.
13. Berte B, Hilfiker G, Russi I, Moccetti F, Cuculi F, Toggweiler S, et al. Pulmonary vein isolation using a higher power shorter duration CLOSE protocol with a surround flow ablation catheter. J Cardiovasc Electrophysiol. 2019;30(11):2199-204. https://doi. org/10.1111/jce.14122.

14. Mattia LD, Crosato M, Indiani S, Causin E, Licciardello C, Squasi PAM, et al. Prospective evaluation of lesion index-guided pulmonary vein isolation technique in patients with paroxysmal atrial fibrillation: 1-year follow-up. J Atr Fibrillation. 2018;10(6):1858. https://doi.org/10.4022/jafib.1858.

15. Pambrun T, Durand C, Constantin M, Masse A, Marra C, Meillet $\mathrm{V}$, et al. High-power $(40-50 \mathrm{~W})$ radiofrequency ablation guided by unipolar signal modification for pulmonary vein isolation. Circ Arrhythm Electrophysiol. 2019;12(6). https://doi.org/10.1161/ circep.119.007304.

16. Bunch TJ, May HT, Bair TL, Crandall BG, Cutler MJ, Mallender $\mathrm{C}$, et al. Long-term outcomes after low power, slower movement versus high power, faster movement irrigated-tip catheter ablation for atrial fibrillation. Heart Rhythm. 2019. https://doi.org/10.1016/j. hrthm.2019.08.001.

17. Chauvin M, Shah DC, Haïssaguerre M, Marcellin L, Brechenmacher $\mathrm{C}$. The anatomic basis of connections between the coronary sinus musculature and the left atrium in humans. Circulation. 2000;101(6):647-52. https://doi.org/10.1161/01.cir. 101.6.647.

18. Wong KCK, Jones M, Sadarmin PP, Bono JD, Qureshi N, Rajappan K, et al. Larger coronary sinus diameter predicts the need for epicardial delivery during mitral isthmus ablation. Ep Europace. 2011;13(4):555-61. https://doi.org/10.1093/europace/eur019.

19. Mao J, Moriarty JM, Mandapati R, Boyle NG, Shivkumar K, Vaseghi M. Catheter ablation of accessory pathways near the coronary sinus: value of defining coronary arterial anatomy. Heart Rhythm. 2015;12(3):508-14. https://doi.org/10.1016/j.hrthm. 2014.11.035.

20. Pothineni NV, Kancharla K, Katoor AJ, Shanta G, Paydak H, Kapa $\mathrm{S}$, et al. Coronary artery injury related to catheter ablation of cardiac arrhythmias: a systematic review. J Cardiovasc Electrophysiol. 2019;30(1):92-101. https://doi.org/10.1111/jce.13764.

21. Mountantonakis SE, Frankel DS, Tschabrunn CM, Hutchinson MD, Riley MP, Lin D, et al. Ventricular arrhythmias from the coronary venous system: prevalence, mapping, and ablation. Heart Rhythm. 2015;12(6):1145-53. https://doi.org/10.1016/j. hrthm.2015.03.009.

22. Meininger GR, Berger RD. Idiopathic ventricular tachycardia originating in the great cardiac vein. Heart Rhythm. 2006;3(4):464-6. https://doi.org/10.1016/j.hrthm.2005.12.021.

Publisher's note Springer Nature remains neutral with regard to jurisdictional claims in published maps and institutional affiliations. 